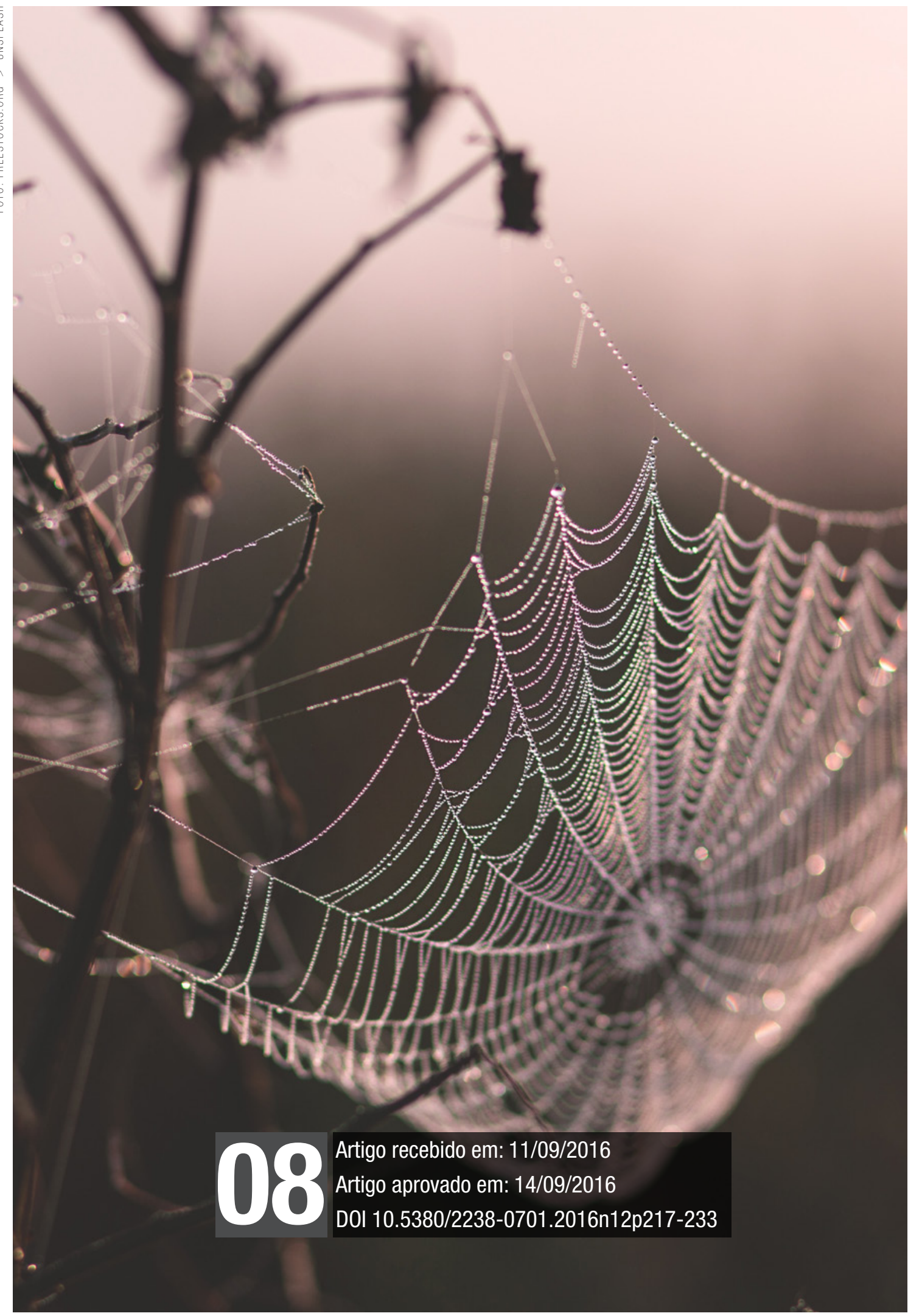


Identidade. Pedagogia digital.Vídeo educativo.

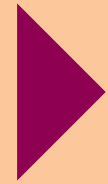




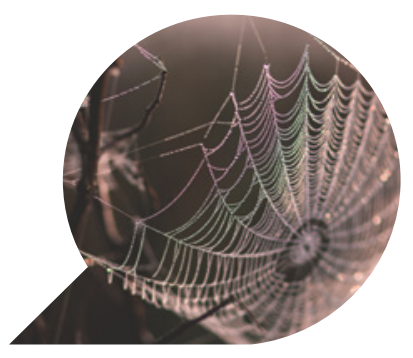

\title{
Vodcasting, \\ quando os estudantes se apresentam ${ }^{1}$
}

\author{
Vodcasting, quand les étudiants se présentent \\ Vodcasting, when students introduce themselves
}

LIA RAQUEL OLIVEIRA ${ }^{2}$

\begin{abstract}
Resumo: Um novo tipo de "cinema" pessoal (vodcasting) está a ser produzido, gerando uma nova economia da imagem na sociedade do conhecimento. Tal acontecimento justifica que se repense o audiovisual na educação, nomeadamente enquanto forma de expressão, na qual a câmara passa para as mãos dos estudantes. Por outro lado, neste mundo digital global, construir a própria identidade tornou-se uma tarefa ainda mais complexa. O vodcasting, pela sua acessibilidade, pode permitir estabelecer novas relações com esta construção assim como novas relações com o saber. Apresentamos um estudo que se debruça sobre uma atividade autobiográfica - vídeos de apresentação de si próprio (um minuto) - na qual 115 vídeos foram produzidos e colocados em portfólios online. Foram pedidas aos estudantes reflexões concisas e os resultados forma analizados bem como os vídeos. A experiência foi considerada muito positiva no que respeita aos conhecimentos e competências adquiridas (multiliteracias) e os estudantes exprimiram um muito alto nível de satisfação pessoal.
\end{abstract}

1 Tradução de Lia Raquel Oliveira

2 Universidade do Minho, Instituto de Educação, Braga, Portugal, lia@ie.uminho.pt 
Palavras-chave: Identidade; Pedagogia universitária digital; vodcasting; vídeo educativo.

Résumé: Un nouveau type de "cinéma " personnel (vodcasting) est en cours de production, donnant lieu à une nouvelle économie de l'image dans la société de la connaissance. Un tel événement justifie que l'on repense l'audiovisuel dans l'éducation, notamment en tant que forme d'expression, où la caméra passe aux mains des étudiants. D’autre part, dans ce monde numérique global, construire son identité propre est devenue une tâche encore plus complexe. Le vodcasting, par son accessibilité, peut permettre d'établir de nouvelles relations à cette construction aussi bien que de nouvelles relations au savoir. Nous présentons une étude portant sur une activité autobiographique - vidéos de présentation de soi-même (une minute) - dans laquelle 115 vidéos ont été produites et placées dans des portfolios en ligne. Des réflexions concises ont été demandées aux étudiants, et leurs résultats ont été analysés ainsi que les vidéos. Lexpérience a été jugée très positive en ce qui concerne les connaissances et les compétences acquises (multiliteraties) et les étudiants ont exprimé un très haut niveau de satisfaction personnelle.

Mots-clés: Identité; Pédagogie universitaire numérique; Vodcasting; Vidéo éducative.

Abstract: A new kind of personal cinema (vodcasting) is being produced, generating a new "image" economy in the Knowledge Society. Such an event justifies a rethinking of audiovisual in education, mainly as a form of expression, where the camera is taken up by the students. Moreover, in this digital global world, building one's own identity has become even more complex. Vodcasting, by its accessability, can bring about new ways of building it, as well as new relationships to knowledge. We present the study of an autobiographical activity - a one minute videogram presentation of oneself in which 115 videograms have been produced and placed in digital portfolios. Brief reflections were demanded from the students, and subjected to content analysis as well as the videograms. The experience was considered to be very positive with knowledge and skills acquired (multiliteracies). A high level of personal satisfaction was declared.

Keywords: Identity; higher education digital technologies; vodcasting, educational video. 


\section{Introdução}

Nos nossos dias, as tecnologias web permitem a circulação massiva de formatos audiovisuais, e cada um pode produzir, cada vez mais facilmente, um cinema pessoal e difundi-lo à escala mundial (YouTube e outras plataformas de partilha), com intenções diversas, gerando uma nova economia da imagem na sociedade do saber. Estas produções vídeo assim como a sua distribuição ou subscrição na Internet, tomam o nome de podcasting ou "podcasting audiovisual" ou, simplesmente, vodcasting. Estas circunstâncias levam a repensar o uso do audiovisual no ensino. Com efeito, de há cerca de dez anos para cá e por todo o mundo, professores preocupados com o sucesso dos seus estudantes experimentaram incorporar o pod/vodcasting nas suas práticas de ensino, no intuito de melhorar o ensino e a aprendizagem.

Interessamo-nos por duas abordagens aos usos do podcasting: a primeira, o vodcasting institucional; a segunda, o vodcasting dirigido e produzido pelos estudantes (nem pelos professores nem por realizadores de cinema pedagógico) como forma de expressão e de aprendizagem. No caso deste estudo, o vodcasting é considerado como possibilidade de mediação e de construção social da realidade (BERGER \& LUCKMANN, 1967) e da identidade, numa época caracterizada pela "fluidez das identidades e declínio da tradição" (GAUNTLET, 2002), na qual "saber como construir socialmente a sua identidade" (ibid.) constitui um dos maiores desafios da existência humana.

Neste texto, apresentamos um estudo exploratório, levado a cabo num contexto universitário, sobre a produção de vodcasts de apresentação pessoal ou "vodcasts autobiográficos".

\section{Os usos do pod/vodcasting}

No domínio da tecnologia educativa, há poucos estudos sobre o vodcasting produzido por estudantes e a maior parte dos existentes não dizem respeito à sua produção por professores. Há autores que encaram os vodcasts enquanto objetos de aprendizagem (GKATZIDOU; PEARSON, 2007); outros como utensílios ao serviço dos professores (BRONW; GREEN, 2007); outros, ainda e na mesma lógica, como armazenamento de aulas/exposições, entrevistas com especialistas, etc. (VAJOCZKI, 2008; OLIVIER; LUCA, 2007). Este último entendimento é o mais generalizado e justifica o interesse de várias universidades 
na utilização de canais YouTube. Os estudos consultados convergem nas mesmas conclusões:

O vodcasting pode melhorar a aprendizagem e aprofundar os níveis de envolvimento e colaboração dos estudantes (Boulos et al., 2006), mas é necessária investigação empírica que compile provas pedagógicas sobre o uso the vodcasts no contexto educacional e sobre o seu potencial em termos de acessibilidade (GKATZIDOU; PEARSON, 2007, p. 327).

Esta investigação empírica tem também interesse dada a natureza atrativa e sedutora desta tecnologia: "Sendo uma excitante e promissora nova tecnologia, está ainda por verificar qual o efeito significante que o video podcasting pode ter na instrução e no design da instrução a longo prazo" (BROWN; GREEN, 2007).

A importância que estes autores atribuem aos efeitos que o podcasting pode vir a ter em longo prazo no design pedagógico é interessante, se considerarmos que as tecnologias são supostas engendrar inovação ao nível pedagógico quando, na realidade, elas apenas são inovadoras no plano técnico e no da difusão (broadcasting). Tal foi o caso da videografia, primeiro analógica e depois digital, e é ainda o caso do vodcasting, integralmente digital, nascido e criado na Internet. O que quer dizer que o vodcasting dos nossos dias é praticado na ausência de qualquer análise ou discussão sobre os efeitos da integração curricular do cinema educativo e da videografia praticados há décadas.

Segundo Bullis (2005), em 2005, os podcasts educativos representavam apenas três por cento dos podcasts disponíveis na Internet e os vodcasts eram praticamente inexistentes. No final do mesmo ano, outro autor avançou a ideia de introduzir o uso do pod/vosdcasting no ensino superior: "bem feito, o podcasting pode revelar aos estudantes, aos professores, ao pessoal, às comunidades - mesmo ao mundo... a humanidade essencial no coração do ensino superior" (CAMPBELL, 2005, p. 42). Nos anos seguintes, o número de publicações que descrevem a utilização de podcasts e de vodcasts aumenta consideravelmente (CARVALHO, 2009; CEBECI; TEKDAL, 2006; LIM, 2006; READ, 2005).

Porém, os estudos convergem igualmente em sublinhar a falta de provas para afirmar que o podcasting tem um impacto significativo no desempenho dos alunos, apesar da atitude positiva a seu respeito 
e da sua aceitação global tanto por estudantes como por professores (VAJOCZKI, 2008).

São, contudo, percebidos aspectos positivos no que concerne ao uso do podcasting em situações de legitimação da participação periférica (LAVE; WENGER, 1991), na observação do saber em ação por especialistas (SCHON, 1983 ; ETHELL; MCMENIMAN, 2000), na projeção pessoal dos estudantes e dos desempenhos dos especialistas que observam (GEE, 2003) e na aprendizagem por resolução de problemas (SAVERY; DUFF, 2001). Outro domínio promissor do podcasting é o da autoscopia em contextos de supervisão (JETNIKOFF, 2007).

Com efeito, o pod/vodcasting tem um potencial educativo importante, constituindo uma situação de aprendizagem convincente, quando utilizado como "veículo de difusão de conteúdo criado por aprendentes" (CHAN, 2006, p. 113). Estes autores sublinham a vantagem de fornecer aos estudantes a oportunidade de fazerem pod/ vodcasts, em particular nas situações de tutoria por pares:

A ideia de ter estudantes na posição de produtores de podcasts encaixa no "modelo de aprendizagem da participação" (Sfard, 1998), por oposição ao modelo da "aquisição", e no qual a aprendizagem significa tornar-se parte de uma comunidade, através da participação e da contribuição de recursos de aprendizagem. Os estudantes são criadores e produtores de conhecimento, ideias e artefactos (Collis \& Moonen, 2001). Ter estudantes de anos avançados a ensinar ou compartilhar os seus conhecimentos e experiências com novos estudantes é consistente com os princípios da tutoria ou ensino por pares (BRONW; CAMPINONE, 1992; BEASLEY, 1997). [...] De acordo com Topping (1996), o mero processo de preparação para ensinar os pares pode melhorar o processamento cognitivo do tutor, do mesmo modo que promove a atenção e motivação para a tarefa, e chama o tutor a revisitar, reorganizar e reintegrar o conhecimento existente (CHAN, 2006, p. 115).

Fornecer aos estudantes a experiência de pod/vodcasting pode estimular uma participação ativa em atividades de aprendizagem socialmente construída e permitir-lhes serem verdadeiramente criadores e produtores de saberes. O pod/vodcasting pode ainda aumentar a partilha entre pares, reaproximando-os à tutoria, favorecendo alternadamente o tutor (que toma uma parte do trabalho do professor como explicar ou representar) e o aprendente. 


\section{Práticas pedagógicas videográficas}

Em todas as disciplinas que ensinamos na universidade integramos sempre, pelo menos, uma atividade de escrita audiovisual (audiovideografia), bem como várias atividades de leitura do audiovisual. Encaramos os vários géneros de filmes disponíveis no ciberespaço eanqunto textos a estudar; para mostrar, fazer pensar, analizar, discutir, como proposta de fórum, etc.

Esta atividade de escrita fílmica - direção/produção/edição de um videograma (vodcast) —, com sinopse, argumanto, guião técnico, persegue os objetivos pedagógicos seguintes: pesquisar informações em diversas fontes, organizar e estruturar as informações tendo em vista a construção do guião técnico (a história do filme); usar uma ferramenta digital de desenho/paginação para construir o storyboard (o filme desenhado); pesquisar elementos gráficos, vídeos e elementos áudio a utilizar na narrativa; planificação da rodagem e da montagem (edição); usar um editor de vídeo digital; e usar outras ferramentas da web 2.0 para proceder ao upload do filme para a Internet (num blogue, sítio pessoal, rede social ou num aplataforma de partilha de vídeos como o YouTube).

\section{Produção de dados e plano metodológico}

Tomando como adquiridas as vantagens da aprendizagem deste cinema educativo na primeira pessoa (OLIVEIRA, 2008), e tendo em mente a necessidade de fornecer aos estudantes uma ocasião de refletir, examinar e exprimir os seus traços de identidade, num processo de "sensibilização" (FREIRE, 2003) desses mesmos traços, introduzimos uma modificação significativa na atividade de videografia no ano letivo 2008/2009: uma atividade individual autobiográfica, cujo tema era a encenação de um videograma de um minuto de autoapresentação.

Os objetivos desta apresentação ficavam ao critério de cada estudante, com múltiplas possibilidades: apresentação a um eventual empregador (espécie de curriculum vitae), apresentação aos colegas de aula ou colegas de trabalho, em suma, o que a imaginação de cada um pudesse criar. No total, foram produzidos 115 videogramas autobiográficos (correspondentes a 115 estudantes), dos quais 100 foram depositados em portfólios digitais, como já era hábito fazermos.

$\mathrm{Na}$ disciplina de Hipertexto (mestrado em ciências da educação, 
Tecnologia Educativa), a atividade foi voluntária (não foi objeto de avaliação das aprendizagens) e foram criados 15 vídeos (em 20 estudantes). O conjunto dos vídeos produzidos ao longo desse ano foram colocados online (quadro 1).

Quadro 1 - Vodcasts criados durante o ano letivo 2008/2009

\begin{tabular}{|l|l|l|l|}
\hline Ciclo & Diploma & Disciplina & $\begin{array}{l}\text { Número de } \\
\text { vodcasts }\end{array}$ \\
\hline $2^{\circ}$ & $\begin{array}{l}\text { Mestrado em Ciências da Educação, } \\
\text { Tecnologia Educativa }\end{array}$ & Hipertexto & 15 \\
\hline $2^{\text {o }}$ & $\begin{array}{l}\text { Mestrado em Educação Mediação Edu- } \\
\text { cacional e Supervisão da Formação }\end{array}$ & $\begin{array}{l}\text { Comunicação e Litera- } \\
\text { cias em Contextos de } \\
\text { Trabalho }\end{array}$ & 17 \\
\hline $2^{\text {o }}$ & $\begin{array}{l}\text { Mestrado em Educação, Educação de } \\
\text { Adultos e Intervenção Comunitária }\end{array}$ & $\begin{array}{l}\text { Aprendizagem em } \\
\text { Rede e Comunicação }\end{array}$ & 33 \\
\hline $1^{\text {o }}$ & Licenciatura em Educação & $\begin{array}{l}\text { Tecnologia e Comuni- } \\
\text { cação Educacional 2 }\end{array}$ & 50 \\
\hline Totais & 4 & 4 & 115 \\
\hline
\end{tabular}

Para além da realização destes vídeos, os estudantes foram convidados a escrever (e colocar nos seus portfólios digitais) uma breve reflexão sobra esta experiência pessoal, indicando dois aspectos positivos e dois aspectos negativos.

Estas reflexões, assim como os vodcasts produzidos, foram submetidas a análise de conteúdo, com a ajuda de uma grelha (grade) de registo/análise concebida para o efeito (quadro 2). 
Quadro 2 - Grade de análise dos vodcasts

\begin{tabular}{|c|c|}
\hline \multicolumn{2}{|l|}{ Contexto : Data : } \\
\hline \multicolumn{2}{|l|}{ Numéro: } \\
\hline \multicolumn{2}{|l|}{ Nome: } \\
\hline \multicolumn{2}{|l|}{ Tema/Título : } \\
\hline Tipo de abordagem & $\begin{array}{l}\text { Exemplos : } \\
\text {-Estilo relatório com entrevistas com... } \\
\text {-Estilo documentário } \\
\text {-Ficção criativa ou inspirada por programa/série/spot televi- } \\
\text { sivo x ou filme y ... } \\
\text {-Narração sequencial cronológica... } \\
\text {-Dramatização... } \\
\text {-Monólogo tipo talking head (registado diretamente na câ- } \\
\text { mara) ou voz off ilustrada... } \\
\text {-Etc. }\end{array}$ \\
\hline Aspectos demonstrados & $\begin{array}{l}\text { Exemplos : } \\
\text {-Cronologia : nascimento (fotos de infầncia com a família, } \\
\text { escola, etc.)... } \\
\text {-Amizades, família, irmãs e irmãos... } \\
\text {-Tempos livres, hobbies } \\
\text {-A importância de alguma coisa ou de alguém ... } \\
\text {-O esforço e a tenacidade... } \\
\text {-As escolhas da vida... originaliddae, envolvimento com... } \\
\text {-Saber fazer alguma coisa de não habitual... } \\
\text {-Desejos... ambições... } \\
\text {-Preocupações pessoais ou sociais... } \\
\text { Etc. }\end{array}$ \\
\hline \multicolumn{2}{|l|}{ Media utilizados } \\
\hline \multicolumn{2}{|l|}{ Foto } \\
\hline \multicolumn{2}{|l|}{ Desenho } \\
\hline \multicolumn{2}{|l|}{ Vídeo } \\
\hline \multicolumn{2}{|l|}{ Música } \\
\hline Locução & \\
\hline
\end{tabular}




\begin{tabular}{|l|l|}
\hline Legendas & \\
\hline Ruídos & \\
\hline Tipo de audiovisual & \\
\hline Diaporama & \\
\hline Vídeo & \\
\hline Misto & \\
\hline Observações: & \\
\hline
\end{tabular}

Nesta grade, o tipo de abordagem está diretamente ligado ao gênero cinematográfico (quando é identificável) e inclui o estilo (formal, informal, humorístico, etc). Os aspectos demonstrados referem-se ao conteúdo propriamente dito, ou seja, as características do sujeito (ou biografia) que foram escolhidas para integrar o filme. Os media utilizados referem-se às formas de expressão empregues (fotografias, desenhos, vídeo, música, locução, legendas, barulhos) e o tipo de audiovisual caracteriza os filmes do ponto de vista das midias usadas (nestas duas secções da grelha, apenas a presença é assinalada. Os aspectos não previstos na grelha são assinalados nas observações. Uma apreciação qualitativa da qualidade global do filme pode ser atribuída (excelente, muito bom, bom, satisfatório, etc.).

Paralelamente, anotamos num diário as situações observadas e os comentários dos estudantes ao longo do processo, aquando da realização e durante as apresentações em aula. Os documentos produzidos foram também equacionados com a taxonomia de podcasts proposta por Carvalho et al. (2008). Em função destas análises, apresentamos agora os resultados mais pertinentes.

\section{Resultados}

Podemos afrmar que estes 115 estudantes implicados nesta experiência pessoal de criação de um videograma de autoapresentação pessoal, em formato vodcast, consideraram que a experiência foi muito positiva, estimulante e motivante (inclusivé para os estudantes que já tinham produzido vídeos noutros contextos e que o referiram nas suas reflexões). Muitos deles consideraram a experiência "única".

Dos aspectos positivos principais destacam-se: a) a aquisição de conhecimentos teóricos e técnicos necessários para produzir e dis- 
ponibilizar um documento desta natureza (conhecimentos sobre o cinema, a linguagem audiovisual e a informática dado o uso de editores de vídeo e da Internet); e b) um nível muito elevado de satisfação pessoal, pelas capacidades criativas que descobriram em si próprios, e que desconheciam, e pelo facto de terem sido capazes de falar de si próprios e de se apresentarem, em suma, porque conseguiram ultrapassar as dificuldades iniciais que se afiguravam numerosas.

Para certas pessoas que não sabiam utilizar os computadores e a Internet ou simplesmente detestavam usar, e para outras que as utilizavam e gostavam de usar, mas nunca tinham sido confrontadas com este tipo de projeto (falar de si mesmo), o sentimento de superação de dificuldade foi preponderante. É necessário não esquecer que a cultura ocidental nem sempre incita à exposição pessoal e muito menos a falar de si mesmo. Em certos vídeos, os autores encontraram outra solução, a saber, pedir a outras pessoas que os apresentassem (membros da família, colegas, amigos) ou colocaram o acento sobre os seus intereses (livros, música, cinema), sobre os seus animais de estimação ou sobre os seus passatempos.

No que respeita aos aspectos negativos, foram mencionadas as limitações de tempo para aprender a trabalhar com as ferramentas e os equipamentos digitais. A dificuldade em conceber o filme e estruturar a narração foi igualmente citada como aspecto negativo: que abordagem adotar, que imagens selecionar, que música, que textos, etc? No entanto, esta dificuldade foi interpretada e percebida como uma vitória em face de um grande desafio.

No que diz respeito aos media usados e ao tipo de audiovisual, a maior parte dos vodcasts adotou o tipo diaporama (identificado como podcast enriquecido na categoria Formato da taxonomia proposta por Carvalho, 2008), colocando em evidência uma série de fotografias com fundo musical, legendas e comentários. Alguns incorporaram pequenos segmentos de vídeo. Poucos vodcasts utilizaram exclusivamente o formato vídeo para a imagem (15/115). Apenas três vídeos se apresentaram sob o formato talking head, ou seja, falado diretamente para a câmara de vídeo. Convém lembrar o quanto a digitalização facilita e permite desenvolver o debate sobre o vídeo enquanto linguagem e como formato. Definitivamente, o podcasting, em sentido alargado e enquanto nova tecnologia compreende documentos que podem ser classificados como audioscriptovisuais, na terminologia criada por Jean Cloutier (1975). 
O tipo de abordagem (gênero) adotado pelos estudantes consistiu, sobretudo, na narração autobiográfica: princípio de vida, a família, a escola, os amigos, até hoje, assim como descrição e caracterização de traços pessoais (as qualidades e os defeitos, as preferências, etc).

Doze destes vodcasts (sobre 115) revelaram escolhas diferentes, oscilando entre a apresentação séria e a valorização de momentos importantes da vida, e a ficção humorística com desfecho inesperado, acentuando o lado lúdico da experiência.

No que concerne as categorias da taxonomia proposta por Carvalho (2008) - o tipo, o formato, a duração, o autor, o estilo, a finalidade - todos os vodcasts produzidos são informativos mas podem ser também considerados como materiais autênticos. Dito de outra forma, a finalidade sendo a apresentação de si mesmo (dar informações sobre si mesmo), com a publicação na Internet estes vodcasts ganham vida própria e adquirem esta dimensão de autenticidade. Tornam-se documentos públicos que representam um indivíduo. Passam a poder ser utilizados por outras pessoas em situações e contextos ilimitados, com intenções e resultados imprevisíveis. Por exemplo, estes vodcasts são já usados como modelo ou referência por outros estudantes a quem a mesma atividade é proposta.

No respeitante à categoria formato, como referimos acima a propósito dos media utilizados e do tipo de audiovisual, apenas 15 documentos podem ser considerados vodcasts, no sentido estrito atribuído pela taxonomia (unicamente vídeo). Os outros vídeos enquadram-se no que a taxonomia designa por "podcast enriquecido" (combinação de imagem com som, diaporama). Porém, apesar da importância do papel de sistematização e clarificação de uma taxonomia, num dado momento, a sua natureza mantêm-se, sem dúvida, flexível e adaptável. Qualquer taxonomia é, necessariamente, um instrumento de trabalho aberto a reformulações que traduzem os avanços no domínio e o questionamneto do objeto ao qual se reporta.

Ainda no quadro desta taxonomia, quanto à categoria "Duração", todos os podcasts são de curta duração (um minuto). Os "Autores" são os próprios estudantes e o "Estilo" foi voluntariamente informal.

\section{Considerações finais}

Esta atividade de vodcasting autobiográfico na primeira pessoa foi muito bem aceite pelos estudantes e afigurou-se como muito grati- 
ficante tanto para os próprios como para a professora. A atividade foi motivante. Os resultados colocam em destaque a viabilidade da hipótese segundo a qual várias atividades criativas de vodcasting poderem ser levadas a cabo por estudantes enquanto herdeiras de certa tradição - pouco difundida e subvalorizada - de utilização do audiovisual (cinema e vídeo) em contexto educativo e numa perspetiva de educação para os media com os media (JACQUINOT, 1981; OLIVEIRA, 2008, 2009).

Ficou claro que a atividade permitiu melhor ver e compreender a construção social da realidade e a construção da identidade. Isto através de um processo partilhado que promoveu, em todos os participantes, a "literacia" no seu sentido mais lato, nomeadamente, no sentido que lhe é atribuído por Paulo Freire $(1989,2003)$ na sua expressão "ler a palavra e ler o mundo" e, mais particularmente aqui, a literacia digital.

Evoluimos numa cultura tecnológica que engendra, como sabemos, os seus próprios excluídos. Os dispositivos tecnológicos (da videovigilância ao Skype) não são simples objetos de consumo. Eles transformam as nossas personalidades - "moldamos as nossas ferramentas e depois são elas quem nos molda"3 (MCLUHAN, 1964).

Refletir sobre a identidade - a nossa forma de nos vermos, de nos conetarmos com os outros e de agir sobre o mundo - utilizando os dispositivos que nos condicionam - no caso, Internet e os media audiovisuais - é, citando Giorgio Agamben (2007), uma estratégia possivel para a luta corpo a corpo quotidiana que travamos com esses dispositivos.

\section{REFERÊNCIAS}

AGAMBEN G. Qu'est-ce qu'un dispositif ? Paris: Rivages Poche, 2007.

BROWN A. \& GREEN T. Podcasting and Video Podcasting : How it Works and How it's Used for Instruction. In: Crawford C. et al. Proceedings of Society for Information Technology and Teacher Education International Conference Chesapeake, 2007. [Disponível em] http://vodcast.editlib.org/p/24857.

BULLIS, K. Podcasting Takes Off. MIT Technology Review, 2005. [Disponível em] http://vodcast.technologyreview.com/web/14821/

CAMPBELL, G. There's Something in the Air : Podcasting in

Education, EDUCAUSE Review, 2005. [Disponível em ]http://vodcast.cblt.soton.

3 No original: we shape our tools and thereafter they shape us. 
ac.uk/multimedia/PDFs08/Podcasting\%20in\%20education.pdf.

CARVALHO, A. A. (org.). Actas do Encontro sobre podcasts. Braga, CIEd: Universidade do Minho, 2009.

CARVAlHO, A. A.; AGUIAR, C.; SANTOS, VODCAST; OLIVEIRA, L. Marques; A. \& Maciel, R. Podcasts in Higher Education: Students' and lecturers' Perspetives. In A. Tatnall \& A. Jones (Eds.). Education and Technology for a Better World. Berlin: Springer, 2009. http://migre.me/nEblq

CEBECI, A; TEKDAL, M. Using Podcasts as Audio Learning Objects. In: Interdisciplinary Journal of Knowledge and Learning Objects, vol. 2, 2006.

[Disponível em ] http://vodcast.ijello.org/Volume2/v2p047-057Cebeci.pdf

CHAN A.; LEE, M. J; VODCAST; MCLOUGHLIN, C. Everyone's learning with podcasting : A Charles Sturt University experience. In: Proceedings of the 23rd annual ascilite conference: Who's learning? Whose technology?, 2006 [Disponível em ]http://vodcast.ascilite.org.au/conferences/sydney06/proceeding/pdf_papers/p171. pdf

CLOUTIER, J. A Era de Emerec ou a Comunicação Audio-scripto-visual na hora dos self-media. Lisboa, 1975.

ETHELL, R.G; MCMENIMAN, M. Unlocking the knowledge in action of an expert practitioner . In: Journal of Teacher Education, 2000. [Disponível em]: 10.1177/002248710005100203

FREIRE, P. A importância do ato de ler em três artigos que se completam. 23 edição, São Paulo, Cortez Editora, 1989.

FREIRE, P. Pedagogia do Oprimido. 36 Edição. Rio de Janeiro, Editora Paz e Terra, 2003.

GAUNTLETT, D. Media, Gender and Identity : An Introduction. London, Routledge, 2002. [Disponível em]: 10.4324/9780203360798

GEE, J.P. What video games have to teach us about learning and literacy. New York, 2003. Palgrave/Macmillan. [Disponível em] 10.1145/950566.950595

GKATZIDOU, S; PEARSON, E. Vodcasting: A case study in adaptability to meet learners'needs and preferences. In : Providing choices for learners and learning, Proceedings Ascilite Singapore, 2007 [Disponível em] http://vodcast.ascilite.org.au/ conferences/singapore07/procs/gkatzidou.pdf.

JACQUINOT, G. On demande toujours des inventeurs. Communications, 1981. DOI : $10.3406 /$ comm.1981.1492 
JETNIKOFF, A.L. From apprentice to performer : using vodcasts to bring English teaching into the tertiary classroom. In: Proceedings 30th HERDSA Annual Conference-Enhancing Higher Education, Theory and Scholarship,Adelaide, Australia, 2007. [Disponível em] http://eprints.qut.edu.au/8995/1/8995.pdf

LAVE, J; WENGER, E. Situated learning : Legitimate peripheral participation. Cambridge, Cambridge University Press, 1991.

\section{LIM, K. Now Hear This - Exploring Podcasting as a Tool in Geography}

Education. Purnell K., Lidstone, J.; Hodgson, S. (orgs.) In: Changes in Geographic Education: Past, Present and Future, Proceedings of the International Geographical Union, 2006.

MCLUHAN, M. Understanding Media: The Extensions of Man. London, Routledge and Kegan Paul, 1964.

OLIVEIRA, L. R. Cinema Educativo e Construção da Realidade. Communication presentée à la conférence Visual Sociology Association International Conference : Space, Time and Image, FLACSO, Buenos Aires, Argentina, 2008.

OLIVEIRA, L. R. Cinema educativo e construção social da realidade : criando identidades através da leitura e da escrita do mundo com o audiovisual. In: Silva B.D., Almeida L.S., Lozano A.B. et Uzquiano M.P. (orgs.), Atas do X Congresso Internacional Galego-Português de Psicopedagogia, Braga, Universidade do Minho, 2009.

OLIVER, R; LUCA, J. Using Mobile Technologies and Podcasts to Enhance Learning Experiences in Lecture-Based University Course Delivery. In: Montgomerie C. et Seale J. (orgs.) Proceedings of World Conference on Educational Multimedia, Hypermedia and Telecommunications. Chesapeake, VA, AACE, 2007 [Disponível em] http://vodcast.editlib.org/p/25859

READ, B. Lectures on the Go. Chronicle of Higher Éducation, 2005.

\section{SAVERY, J. R.; DUFFY, T. M. Problem based learning : An instructional} model and its constructivist framework. Centre for Research on Learning and Technology, Technical Report No. 16-01, Indiana University, 2001.

SCHON, D. A. The reflective practitioner : How professionals think in action. New York, Basic Books. 1983. DOI : 10.1080/07377366.1986.10401080

THE NEW LONDON GROUP. A Pedagogy of Multiliteracies : Designing Social Futures. Harward Educational Review, vol. 66, 1996. 
VAJOCZKI, S.; WATT, S.; MARQUIS, N. Vodcasts: Are they an effective tool to enhance student learning ? A Case Study from McMaster University,

Hamilton Canada. In: Proceedings of World Conference on Educational Multimedia, Hypermedia and Telecommunications. Chesapeake, VA, AACE, 2008 [Disponível em] http://vodcast.editlib.org/p/29053 